\title{
Algebraic Approach to Exact Solution of the $(2+1)$-Dimensional Dirac Oscillator in the Noncommutative Phase Space
}

\author{
H. Panahi and A. Savadi \\ Department of Physics, University of Guilan, Rasht 41635-1914, Iran \\ Correspondence should be addressed to H. Panahi; t-panahi@guilan.ac.ir \\ Received 28 April 2017; Revised 6 August 2017; Accepted 11 September 2017; Published 17 October 2017 \\ Academic Editor: Elias C. Vagenas
}

Copyright (C) 2017 H. Panahi and A. Savadi. This is an open access article distributed under the Creative Commons Attribution License, which permits unrestricted use, distribution, and reproduction in any medium, provided the original work is properly cited. The publication of this article was funded by SCOAP

We study the $(2+1)$-dimensional Dirac oscillator in the noncommutative phase space and the energy eigenvalues and the corresponding wave functions of the system are obtained through the $\mathrm{sl}(2)$ algebraization. It is shown that the results are in good agreement with those obtained previously via a different method.

\section{Introduction}

Study on Dirac oscillator as an important potential has attracted a lot of attention and has found many physical applications in various branches of physics [1-6]. The Dirac oscillator was introduced for the first time by Itô et al. [7], in which the momentum $\vec{p}$ in Dirac equation is replaced by $\vec{p}-i m_{0} \omega \widetilde{\beta} \vec{r}$, where $\vec{r}$ is the position vector and $m_{0}, \omega$, and $\widetilde{\beta}$ are the mass of particle, the frequency of the oscillator, and the usual Dirac matrices, respectively. Similar system was studied by Moshinsky and Szczepaniak [8], who gave it the name of Dirac oscillator; due to the nonrelativistic limit, it becomes a simple harmonic oscillator with strong spin-orbit coupling term. In quantum optics and for $(2+1)$-dimension space, it is seen that the Dirac oscillator system can be mapped into Anti-Jaynes-Cummings model [9-11] which describes the atomic transitions in a two-level system. In $[5,12]$, it has been shown that the Dirac oscillator interaction can be interpreted as the interaction of the anomalous magnetic moment with a linear electric field. Also the electromagnetic potential associated with the Dirac oscillator interaction has been found by Benitez et al. in [13].

On the other hand in recent decades, the noncommutativity has become an extremely active area of research such as in string theories, quantum field theories, and quantum mechanics [14-24]. For quantum systems in noncommutative space, it is seen that assuming the noncommutativity may be a result of quantum gravity effects, also it is a fruitful theoretical laboratory where we can get some insight on the consequences of noncommutativity in field theory by using standard calculation techniques of quantum mechanics. The study on Dirac equation in noncommutative phase space has also attracted much attention in recent years [25-31]. For example, study on the relativistic Landau levels of Dirac equation in $(2+1)$-dimensional noncommutative phase space has been shown in [28] and one can see an exact mapping of this relativistic model to the AJC model. Also in [25], it is seen that, for Landau problem in two-dimensional noncommutative phase space, the equation of motion of a harmonic oscillator is similar to the equation of motion of a particle in a constant magnetic field and in the lowest Landau level. The energy gap of Dirac oscillator in a noncommutative phase space changes by noncommutative effect [25]. A noncommutative description of graphene, which consists of a Dirac equation for massless Dirac fermions plus noncommutative corrections, has been studied in [27] and it has been shown that the momentum noncommutativity affects the energy levels of grapheme.

Since the appearance of quantum mechanics, considerable efforts have been devoted to obtaining exact solutions of the relativistic and nonrelativistic wave equations, using different methods and techniques [32-36]. Now in this paper, we study the Dirac oscillator in noncommutative phase space 
within the framework of representation theory of the sl(2) Lie algebra.

A quantum system is exactly solvable (ES) if all the eigenvalues and the corresponding eigenfunctions can be calculated in an exact analytical manner. In contrast, a quantum system is quasi-exactly solvable (QES) if only a finite number of eigenvalues and eigenfunctions can be determined exactly [37-40]. In the case of ES models, the Hamiltonian of the system can be diagonalized completely due to the fact that algebraic symmetry is complete, but in the case of QES models, the Hamiltonian is only block diagonalized. Such a finite block can always be diagonalized, which yields a finite part of the spectrum algebraically.

This paper is organized as follows. In Section 2, based on [31], we briefly review the noncommutative phase space on (2 +1 )-dimensional Dirac oscillator. Section 3 is devoted to $\operatorname{sl}(2)$ algebraization of Dirac oscillator in the noncommutative phase space. We obtain the energy eigenvalues and the corresponding wave functions through the $\mathrm{sl}(2)$ representation and show that our results are in good agreement with the results of [31]. In Section 4, we present the conclusion.

\section{Review on $(2+1)$-Dimensional Dirac Oscillator in the Noncommutative Phase Space}

According to [31], the noncommutative phase space is characterized by the fact that their coordinate operators satisfy the equation

$$
\begin{aligned}
& {\left[x_{i}^{(\mathrm{NC})}, x_{j}^{(\mathrm{NC})}\right]=i \widetilde{\theta}_{i j},} \\
& {\left[p_{i}^{(\mathrm{NC})}, p_{j}^{(\mathrm{NC})}\right]=i \bar{\theta}_{i j},} \\
& {\left[x_{i}^{(\mathrm{NC})}, p_{j}^{(\mathrm{NC})}\right]=i \hbar \delta_{i j},}
\end{aligned}
$$

where $\widetilde{\theta}_{i j}$ and $\bar{\theta}_{i j}$ are an antisymmetric tensor of space dimension. By using Bopp's shift method, the two-dimensional noncommutative phase space can be considered as [29]

$$
\begin{aligned}
& x^{(\mathrm{NC})}=x-\frac{\tilde{\theta}}{2 \hbar} p_{y}, \\
& y^{(\mathrm{NC})}=y+\frac{\tilde{\theta}}{2 \hbar} p_{x}, \\
& p_{x}^{(\mathrm{NC})}=p_{x}+\frac{\bar{\theta}}{2 \hbar} y, \\
& p_{y}^{(\mathrm{NC})}=p_{y}-\frac{\bar{\theta}}{2 \hbar} x .
\end{aligned}
$$

The Dirac oscillator is also described by the following Hamiltonian [8]:

$$
\widehat{H}=c \widehat{\alpha} \cdot\left(\vec{p}-i m_{0} \omega \widetilde{\beta r}\right)+\widetilde{\beta} m_{0} c^{2}
$$

where, in two-dimensional noncommutative space, it is transformed as

$$
\begin{aligned}
& \left\{c \alpha_{x}\left(p_{x}^{(\mathrm{NC})}-i m_{0} \omega \tilde{\beta} x^{(\mathrm{NC})}\right)\right. \\
& \left.\quad+c \alpha_{y}\left(p_{y}^{(\mathrm{NC})}-i m_{0} \omega \widetilde{\beta} y^{(\mathrm{NC})}\right)+\widetilde{\beta} m_{0} c^{2}\right\} \psi_{D} \\
& \quad=E_{\mathrm{NC}} \psi_{D} .
\end{aligned}
$$

By considering the Pauli matrices as the representation of Dirac matrices in $(2+1)$-dimension, that is,

$$
\begin{aligned}
& \alpha_{x}=\sigma_{x}=\left(\begin{array}{ll}
0 & 1 \\
1 & 0
\end{array}\right), \\
& \alpha_{y}=\sigma_{y}=\left(\begin{array}{cc}
0 & -i \\
i & 0
\end{array}\right), \\
& \widetilde{\beta}=\left(\begin{array}{cc}
1 & 0 \\
0 & -1
\end{array}\right)
\end{aligned}
$$

and $\psi_{D}=\left(\begin{array}{ll}\psi_{1} & \psi_{2}\end{array}\right)^{T},(4)$ becomes

$$
\left(\begin{array}{cc}
m_{0} c^{2} & c p_{-} \\
c p_{+} & -m_{0} c^{2}
\end{array}\right)\left(\begin{array}{l}
\psi_{1} \\
\psi_{2}
\end{array}\right)=E_{\mathrm{NC}}\left(\begin{array}{l}
\psi_{1} \\
\psi_{2}
\end{array}\right)
$$

where $p_{-}, p_{+}$are as follows:

$$
\begin{aligned}
p_{-} & =p_{x}^{(\mathrm{NC})}-i p_{y}^{(\mathrm{NC})}+i m_{0} \omega\left(x^{(\mathrm{NC})}-i y^{(\mathrm{NC})}\right) \\
& =\rho_{1}\left(p_{x}-i p_{y}\right)+i m_{0} \omega \rho_{2}(x-i y), \\
p_{+} & =p_{x}^{(\mathrm{NC})}+i p_{y}^{(\mathrm{NC})}-i m_{0} \omega\left(x^{(\mathrm{NC})}+i y^{(\mathrm{NC})}\right) \\
& =\rho_{1}\left(p_{x}+i p_{y}\right)-i m_{0} \omega \rho_{2}(x+i y),
\end{aligned}
$$

with

$$
\begin{aligned}
& \rho_{1}=1+\frac{m_{0} \omega}{2 \hbar} \tilde{\theta}, \\
& \rho_{2}=1+\frac{\bar{\theta}}{2 m_{0} \omega \hbar} .
\end{aligned}
$$

After some calculations, it is easy to see that (6) gives

$$
\begin{aligned}
& \left\{c^{2} p_{-} p_{+}-\left(E_{\mathrm{NC}}^{2}-m_{0}^{2} c^{4}\right)\right\} \psi_{1}=0 \\
& \left\{c^{2} p_{+} p_{-}-\left(E_{\mathrm{NC}}^{2}-m_{0}^{2} c^{4}\right)\right\} \psi_{2}=0
\end{aligned}
$$

Similar to $[31,41]$ and by defining $p_{x}=p \cos \theta, p_{y}=p \sin \theta$, and $p^{2}=p_{x}^{2}+p_{y}^{2}$, it is seen that the (7) transform into

$$
\begin{aligned}
& p_{-}=e^{-i \theta}\left\{\rho_{1} p-\lambda\left(\frac{\partial}{\partial p}-\frac{i}{p} \frac{\partial}{\partial \theta}\right)\right\}, \\
& p_{+}=e^{i \theta}\left\{\rho_{1} p+\lambda\left(\frac{\partial}{\partial p}+\frac{i}{p} \frac{\partial}{\partial \theta}\right)\right\},
\end{aligned}
$$


where

$$
\lambda=\left(1+\frac{\bar{\theta}}{2 m_{0} \omega \hbar}\right) m_{0} \omega \hbar .
$$

Substituting (11) into (9) and by considering $\psi_{1}(p, \theta)=$ $f(p) e^{i m \theta}$, we have

$$
\begin{gathered}
\left(\frac{d^{2} f(p)}{d p^{2}}+\frac{1}{p} \frac{d f(p)}{d p}-\frac{m^{2}}{p^{2}} f(p)\right) \\
+\left(\kappa^{2}-k^{2} p^{2}\right) f(p)=0
\end{gathered}
$$

where

$$
\begin{aligned}
& \kappa^{2}=\frac{2 \lambda \rho_{1}(m+1)+\varepsilon}{\lambda^{2}}, \\
& k^{2}=\frac{\rho_{1}^{2}}{\lambda^{2}},
\end{aligned}
$$

with

$$
\varepsilon=\frac{E_{\mathrm{NC}}^{2}-m_{0}^{2} c^{4}}{c^{2}} .
$$

Here it is necessary to point out that, in the literature, there are many different methods for solving quantum models exactly [32-36]. Boumali and Hassanabadi [31] solved this problem analytically and obtained the exact solutions in terms of the hypergeometric functions. Within the present study, we intend to illustrate the exact solvability of the problem through the $s l(2)$ algebraization [39]. To do a comprehensive analysis, we need to obtain the more general solution of the problem. This is the main advantage of the present method. In fact, by using the theory of representation space, we construct the general matrix equation of the problem and calculate the closed-form expressions for energies and eigenfunctions. Accordingly, we can quickly obtain exact solutions of any arbitrary state $n$, without any cumbersome numerical procedure or a complicated analytical one in determining the solutions of the higher states. It seems that the method is computationally much simpler than other analytical methods.

Hence in the next section, we solve (13), by Lie algebraic approach related to representation theory of sl(2) Lie group. Similar procedure can be also done on (10) for the $\psi_{2}$ spinor wave function.

\section{Lie Algebraic Solution of the Dirac Oscillator in the Noncommutative Phase Space}

In this section, for solving (13) by the sl(2) Lie algebra representation, we first use similarity transformation as $f(p)=p^{-1 / 2} R(p)$, such that the first-order derivative of the differential equation (13) can be eliminated, and it is reduced to a Schrödinger-type operator as

$$
\left(\frac{d^{2}}{d p^{2}}+\frac{1 / 4-m^{2}}{p^{2}}-\kappa^{2}-k^{2} p^{2}\right) R(p)=0 .
$$

Extracting the asymptotic behavior of the wavefunction at the origin and infinity, using the transformation

$$
R(p)=p^{\gamma} e^{\alpha p^{2}} F(p),
$$

where $\alpha<0$ (to be physically meaningful) and $\gamma$ are parameters to be determined later by the exact solvability conditions (see (26)), (16) is converted to

$$
\begin{aligned}
& \frac{d^{2} F(p)}{d p^{2}}+\left(\frac{2 \gamma}{p}+4 \alpha p\right) \frac{d F(p)}{d p}+\left(\left(4 \alpha^{2}-k^{2}\right) p^{2}\right. \\
& \left.+\frac{\gamma(\gamma-1)+1 / 4-m^{2}}{p^{2}}+4 \alpha \gamma+2 \alpha+\kappa^{2}\right) F(p) \\
& =0 .
\end{aligned}
$$

Using the change of variable $r=k p^{2}$ we get

$$
\begin{aligned}
& r \frac{d^{2} F}{d r^{2}}+\left(\frac{2 \alpha}{k} r+\gamma+\frac{1}{2}\right) \frac{d F}{d r}+\left[\left(\frac{\alpha^{2}}{k^{2}}-\frac{1}{4}\right) r\right. \\
& \left.+\frac{\gamma(\gamma-1)+1 / 4-m^{2}}{4 r}+\frac{\alpha \gamma}{k}+\frac{\alpha}{2 k}+\frac{\kappa^{2}}{4 k}\right] F=0 .
\end{aligned}
$$

According to [38], in one dimension, the only Lie algebra of the first-order differential operators, which possesses finitedimensional representations, is the sl(2) Lie algebra. Hence we consider the following realization of the operators:

$$
\begin{aligned}
& J_{n}^{+}=r^{2} \frac{d}{d r}-n r, \\
& J_{n}^{0}=r \frac{d}{d r}-\frac{n}{2}, \\
& J_{n}^{-}=\frac{d}{d r},
\end{aligned}
$$

which satisfy the $\mathrm{sl}(2)$ commutation relations

$$
\begin{aligned}
& {\left[J_{n}^{+}, J_{n}^{-}\right]=-2 J_{n}^{0},} \\
& {\left[J_{n}^{ \pm}, J_{n}^{0}\right]=\mp J_{n}^{ \pm}}
\end{aligned}
$$

and preserve the $(n+1)$-dimensional linear space of polynomials with finite order

$$
P_{n+1}=\left\langle 1, r, r^{2}, \ldots, r^{n}\right\rangle .
$$

The most general second-order differential equation which preserves the space $P_{n+1}$ can be also represented as a quadratic combination of the sl(2) generators as

$$
H=\sum_{a, b=0, \pm} C_{a b} J_{n}^{a} J_{n}^{b}+\sum_{a=0, \pm} C_{a} J_{n}^{a}+C,
$$

where $C_{a b}, C_{a}$, and $C$ are real parameters. Substituting (20) into (23) yields the following differential form:

$$
H=P_{4}(r) \frac{d^{2}}{d r^{2}}+P_{3}(r) \frac{d}{d r}+P_{2}(r),
$$


where $P_{i}(r)$ are polynomials of degree $i$

$$
\begin{aligned}
P_{4}(r)= & C_{++} r^{4}+C_{+0} r^{3}+C_{+-} r^{2}+C_{0-} r+C_{--}, \\
P_{3}(r)= & C_{++}(2-2 n) r^{3}+\left(C_{+}+C_{+0}\left(1-\frac{3 n}{2}\right)\right) r^{2} \\
& +\left(C_{0}-n C_{+-}\right) r+\left(C_{-}-\frac{n}{2} C_{0-}\right), \\
P_{2}(r)= & C_{++} n(n-1) r^{2}+\left(\frac{n^{2}}{2} C_{+0}-n C_{+}\right) r \\
& +\left(C-\frac{n}{2} C_{0}\right) .
\end{aligned}
$$

Comparing (24) with (19) gives

$$
\begin{aligned}
C_{++} & =C_{+0}=C_{+-}=C_{--}=C_{+}=0, \\
\alpha & =-\frac{k}{2}, \\
\gamma & =m+\frac{1}{2}, \\
C_{0-} & =1, \\
C_{0} & =-1,
\end{aligned}
$$

$$
\begin{aligned}
C_{-} & =\frac{n}{2}+m+1, \\
C & =\frac{\kappa^{2}}{4 k}-\frac{1}{2}(m+n+1) .
\end{aligned}
$$

From (23) and (26), the Lie algebraic form of the Hamiltonian is written as

$$
\begin{aligned}
H= & J_{n}^{0} J_{n}^{-}-J_{n}^{0}+\left(\frac{n}{2}+m+1\right) J_{n}^{-} \\
& +\left(\frac{\kappa^{2}}{4 k}-\frac{1}{2}(m+n+1)\right),
\end{aligned}
$$

which implies that this operator is ES, due to the absence of the terms of positive grading. Therefore, the operator $H$ preserves the finite-dimensional representation space of the algebra $\mathrm{sl}(2)$ as

$$
F(r)=\sum_{j=0}^{n} a_{j} r^{j}, \quad n=0,1,2, \ldots
$$

Hence, using (27) and (28) and doing some calculations, the following matrix equation can be obtained:

$$
\begin{aligned}
& \begin{array}{c}
\left(\begin{array}{ccc}
\frac{\kappa^{2}}{4 k}-\frac{1}{2}(m+1) & 0 \\
0 & \frac{\kappa^{2}}{4 k}-\frac{1}{2}(m+1)-1 & 2+2(m+1) \\
0 & \vdots & \ddots \\
\vdots & 0
\end{array}\right. \\
=0, \quad \ldots \\
\frac{\kappa^{2}}{4 k}-\frac{1}{2}(m+1)-n=0, \quad n=0,1,2, \ldots
\end{array} \\
& E_{\mathrm{NC}_{n}} \\
& = \pm m_{0} c^{2} \sqrt{1+4 n\left(1+\frac{m_{0} \omega}{2 \hbar} \tilde{\theta}\right)\left(1+\frac{\bar{\theta}}{2 m_{0} \omega \hbar}\right) \frac{\omega \hbar}{m_{0} c^{2}}},
\end{aligned}
$$

which is the same as the energy relation in [31].
Also from (29) the expansion coefficients $a_{m}$ satisfy the following two-term recursion relation:

$$
\begin{aligned}
& (j(j+1)+(j+1)(m+1)) a_{j+1} \\
& +\left(\frac{\kappa^{2}}{4 k}-\frac{1}{2}(m+1)-j\right) a_{j}=0
\end{aligned}
$$

with the boundary conditions $a_{-1}=0$ and $a_{n+1}=0$.

Before we proceed further, it would be well to note that (31) may or may not possess real eigenvalues depending on the choice of noncommutative parameters $\widetilde{\theta}$ and $\bar{\theta}$ to be positive or negative values. Of course, it is not our aim to 
study here, but one can see a similar study in [42], where the Dirac oscillator in $2+1$-dimensional noncommutative space has been studied for the cases of positive and negative noncommutative parameter. Hence it seems that the results can be used to study the connection with non-Hermitian quantum mechanics. Also, the possible case of imaginary energy gives useful clue regarding the lack of bound-states in the spectroscopy of relativistic fermions.

Therefore for a given $n$, the energy eigenvalue is obtained from (31) and the corresponding unnormalized wave function can be obtained from (6) as

$$
\psi_{D} \equiv \psi_{n, m}(p, \theta)=\left(\begin{array}{l}
\psi_{1}(p, \theta) \\
\psi_{2}(p, \theta)
\end{array}\right)
$$

$$
=\left(\begin{array}{c}
1 \\
\frac{c p_{+}}{E+m_{0} c^{2}}
\end{array}\right) \psi_{1}(p, \theta),
$$

where

$$
\psi_{1}(p, \theta)=p^{m} e^{-(k / 2) p^{2}} e^{i m \theta} \sum_{j=0}^{n} a_{j} r^{j},
$$

with $r=k p^{2}$. Now for clarifying that the wave function obtained from this method is in good agreement with the results of [31], we calculate it for $n=2$. According to (28) and (32), the three-dimensional invariant subspace is obtained as

$$
\begin{aligned}
F(r) & =a_{0}+a_{1} r+a_{2} r^{2} \\
& =a_{0}\left[1+\frac{\left(-\kappa^{2} / 4 k+(1 / 2)(m+1)\right)}{m+1}\left(k p^{2}\right)+\frac{\left(-\kappa^{2} / 4 k+(1 / 2)(m+1)\right)\left(-\kappa^{2} / 4 k+(1 / 2)(m+1)+1\right)}{(m+1)(2+2(m+1))}\left(k p^{2}\right)^{2}\right],
\end{aligned}
$$

where by using the definition of the confluent hypergeometric function for $n=2$ we have

$$
F(r)=a_{01} F_{1}\left(-n, m+1 ; k p^{2}\right) .
$$

The above equation is exactly identical to equation (32) in [31] and so for other values of $n$ and after some calculations, one can show that $F(r)$ can be written in terms of the confluent hypergeometric function.

\section{Conclusions}

Using the Lie algebraic approach, we have solved the Dirac oscillator in $(2+1)$ dimension in the framework of noncommutative phase space. We have obtained the energy eigenvalues and the corresponding wave functions through the $\mathrm{sl}(2)$ Lie algebra representation. We have also shown that our results are in good agreement with the results in [31]. It is seen that the Lie algebraic approach is a powerful method for reproducing the exact analytical results.

\section{Conflicts of Interest}

The authors declare that they have no conflicts of interest.

\section{References}

[1] A. Bermudez, M. A. Martin-Delgado, and A. Luis, "Chirality quantum phase transition in the Dirac oscillator," Physical Review A, vol. 77, no. 6, Article ID 063815, 2008.

[2] N. Ferkous and A. Bounames, "Energy spectrum of a $2 \mathrm{D}$ Dirac oscillator in the presence of the Aharonov-Bohm effect," Physics Letters A, vol. 325, no. 1, pp. 21-29, 2004.

[3] V. M. Villalba, "Exact solution of the two-dimensional Dirac oscillator," Physical Review A, vol. 49, no. 1, 586 pages, 1994.
[4] A. Bermudez, M. A. Martin-Delgado, and E. Solano, "Exact mapping of the $2+1$ Dirac oscillator onto the Jaynes-Cummings model: Ion-trap experimental proposal," Physical Review A, vol. 76, no. 4, Article ID 041801, 2007.

[5] M. Moreno and A. Zentella, "Covariance, CPT and the FoldyWouthuysen transformation for the Dirac oscillator," Journal of Physics A: Mathematical and General, vol. 22, no. 17, L821 pages, 1989.

[6] R. de Lima Rodrigues, "On the Dirac oscillator," Physics Letters $A$, vol. 372, no. 15, pp. 2587-2591, 2008.

[7] D. Itô, K. Mori, and E. Carriere, "An example of dynamical systems with linear trajectory," Il Nuovo Cimento A, vol. 51, no. 4, pp. 1119-1121, 1967.

[8] M. Moshinsky and A. Szczepaniak, "The Dirac oscillator," Journal of Physics A: Mathematical and General, vol. 22, no. 17, L817 pages, 1989.

[9] E. T. Jaynes and F. W. Cummings, "Comparison of quantum and semiclassical radiation theories with application to the beam maser," Proceedings of the IEEE, vol. 51, no. 1, pp. 89-109, 1963.

[10] J. Larso, "Dynamics of the Jaynes-Cummings and Rabi models: old wine in new bottles," Physica Scripta, vol. 76, no. 2, 146 pages, 2007.

[11] L. Allen and J. H. Eberly, Optical Resonance and Two Level Atoms, Dover Publications, Mineola, New York, USA, 1987.

[12] R. P. Martinez-y-Romero and A. L. Salas-Brito, "Conformal invariance in a Dirac oscillator," Journal of Mathematical Physics, vol. 33, no. 5, 1831 pages, 1992.

[13] J. Benitez, R. P. Martnez y Romero, H. N. Núez-Yépez, and A. L. Salas-Brito, "Solution and hidden supersymmetry of a Dirac oscillator," Physical Review Letters, vol. 64, 1643, no. 14, 1990.

[14] N. Seiberg and E. Witten, "String theory and noncommutative geometry," Journal of High Energy Physics, vol. 1999, no. 09, article 032, 1999.

[15] A. Connes, M. R. Douglas, and A. Schwarz, "Noncommutative geometry and Matrix theory," Journal of High Energy Physics, vol. 1998, 003, no. 02, 1998. 
[16] M. R. Douglas and N. A. Nekrasov, "Noncommutative field theory," Reviews of Modern Physics, vol. 73, no. 4, 977 pages, 2001.

[17] C. S. Chu and P. M. Ho, "Non-commutative open string and Dbrane," Nuclear Physics B, vol. 550, no. 1-2, pp. 151-168, 1999.

[18] C.-S. Chu and P.-M. Ho, "Constrained quantization of open string in background B field and non-commutative D-brane," Nuclear Physics B, vol. 568, no. 1-2, pp. 447-456, 2000.

[19] F. Ardalan, H. Arfaei, and M. M. Sheikh-Jabbari, "Dirac quantization of open strings and noncommutativity in branes," Nuclear Physics B, vol. 576, no. 1-3, pp. 578-596, 2000.

[20] J. Jing and Z.-W. Long, "Open string in the constant B-field background," Physical Review D, vol. 72, no. 12, 126002 pages, 2005.

[21] I. Hinchliffe, N. Kersting, and Y. L. Ma, "Review of the phenomenology of noncommutative geometry", International Journal of Modern Physics A. Particles and Fields; Gravitation; Cosmology, vol. 19, no. 2, 179 pages, 2004.

[22] S. Minwalla, M. V. Raamsdonk, and N. Seiberg, "Noncommutative perturbative dynamics," Journal of High Energy Physics, vol. 2000, 020, no. 02, 2000.

[23] M. V. Raamsdonk and N. Seiberg, "Comments on noncommutative perturbative dynamics," Journal of High Energy Physics, vol. 2000, 035, no. 03, 2000.

[24] R. Gopakumar, S. Minwalla, and A. Strominger, "Noncommutative solitons," Journal of High Energy Physics, vol. 2000, 020, no. $05,2000$.

[25] S. Cai, T. Jing, G. Guo, and R. Zhang, "Dirac oscillator in noncommutative phase space," International Journal of Theoretical Physics, vol. 49, no. 8, pp. 1699-1705, 2010.

[26] O. Bertolami and R. Queiroz, "Phase-space noncommutativity and the Dirac equation," Physics Letters. A, vol. 375, no. 46, pp. 4116-4119, 2011.

[27] Z.-Y. Luo, Q. Wang, X. Li, and J. Jing, "Dirac oscillator in noncommutative phase space and (anti)-Jaynes-Cummings models," International Journal of Theoretical Physics, vol. 51, no. 7, pp. 2143-2151, 2012.

[28] B. P. Mandal and S. K. Rai, "Noncommutative Dirac oscillator in an external magnetic field," Physics Letters A, vol. 376, no. 36, pp. 2467-2470, 2012.

[29] C. Bastos, O. Bertolami, N. C. Dias, and J. N. Prata, "Noncommutative graphene," International Journal of Modern Physics A, vol. 28, no. 16, Article ID 1350064, 13 pages, 2013.

[30] O. Panella and P. Roy, "Quantum phase transitions in the noncommutative Dirac oscillator," Physical Review A, vol. 90, no. 4, Article ID 042111, 2014.

[31] A. Boumali and H. Hassanabadi, "Exact solutions of the $(2+$ 1)-dimensional dirac oscillator under a magnetic field in the presence of a minimal length in the non-commutative phase space," Zeitschrift für Naturforschung A - A Journal of Physical Sciences, vol. 70, no. 8, 619 pages, 2015.

[32] H. Ciftci, R. L. Hall, and N. Saad, "Asymptotic iteration method for eigenvalue problems," Journal of Physics A: Mathematical and General, vol. 36, no. 47, 11807 pages, 2003.

[33] S. H. Dong, D. Morales, and J. Garcia-Ravelo, "Exact quantization rule and its applications to physical potentials," International Journal of Modern Physics E, vol. 16, no. 1, p. 189, 2007.

[34] G. F. Wei and S. H. Dong, "Algebraic approach to energy spectra of the Scarf type and generalized Pöschl-Teller potentials," Canadian Journal of Physics, vol. 89, no. 12, pp. 1225-1231, 2011.
[35] H. Hassanabadi, S. Zarrinkamar, and A. A. Rajabi, "Exact solutions of $D$-dimensional Schrödinger equation for an energydependent potential by NU method," Communications in Theoretical Physics, vol. 55, no. 4, pp. 541-544, 2011.

[36] H. Hassanabadi, A. A. Rajabi, and S. Zarrinkamar, "Cornell and kratzer potentials within the semirelativistic treatment," Modern Physics Letters A, vol. 27, no. 10, Article ID 1250057, 2012.

[37] A. G. Ushveridze, "Lie-algebraic approach to the problem of quasi-exact solubility in quantum mechanics," Modern Physics Letters A. Particles and Fields, Gravitation, Cosmology and Nuclear Physics, vol. 5, no. 23, pp. 1891-1899, 1990.

[38] A. V. Turbiner, "Quasi-exactly-solvable problems and $\mathrm{sl}(2)$ algebra," Communications in Mathematical Physics, vol. 118, no. 3, pp. 467-474, 1988.

[39] A. V. Turbiner, "Lie-algebras and linear operators with invariant subspaces," Contemporary Mathematics, vol. 160, p. 263, 1994.

[40] D. Gómez-Ullate, N. Kamran, and R. Milson, "Quasi-exact solvability beyond the $\mathrm{sl}(2)$ algebraization," Physics of Atomic Nuclei, vol. 70, no. 3, pp. 520-528, 2007.

[41] L. Menculini, O. Panella, and P. Roy, "Exact solutions of the (2 +1 ) dimensional Dirac equation in a constant magnetic field in the presence of a minimal length," Physical Review D, vol. 87, no. 6, Article ID 065017, 2013.

[42] Y.-Q. Luo, Y. Cui, Z.-W. Long, and J. Jing, "2 + 1 Dimensional noncommutative dirac oscillator and (anti)-jaynes-cummings models," International Journal of Theoretical Physics, vol. 50, no. 10, pp. 2992-3000, 2011. 

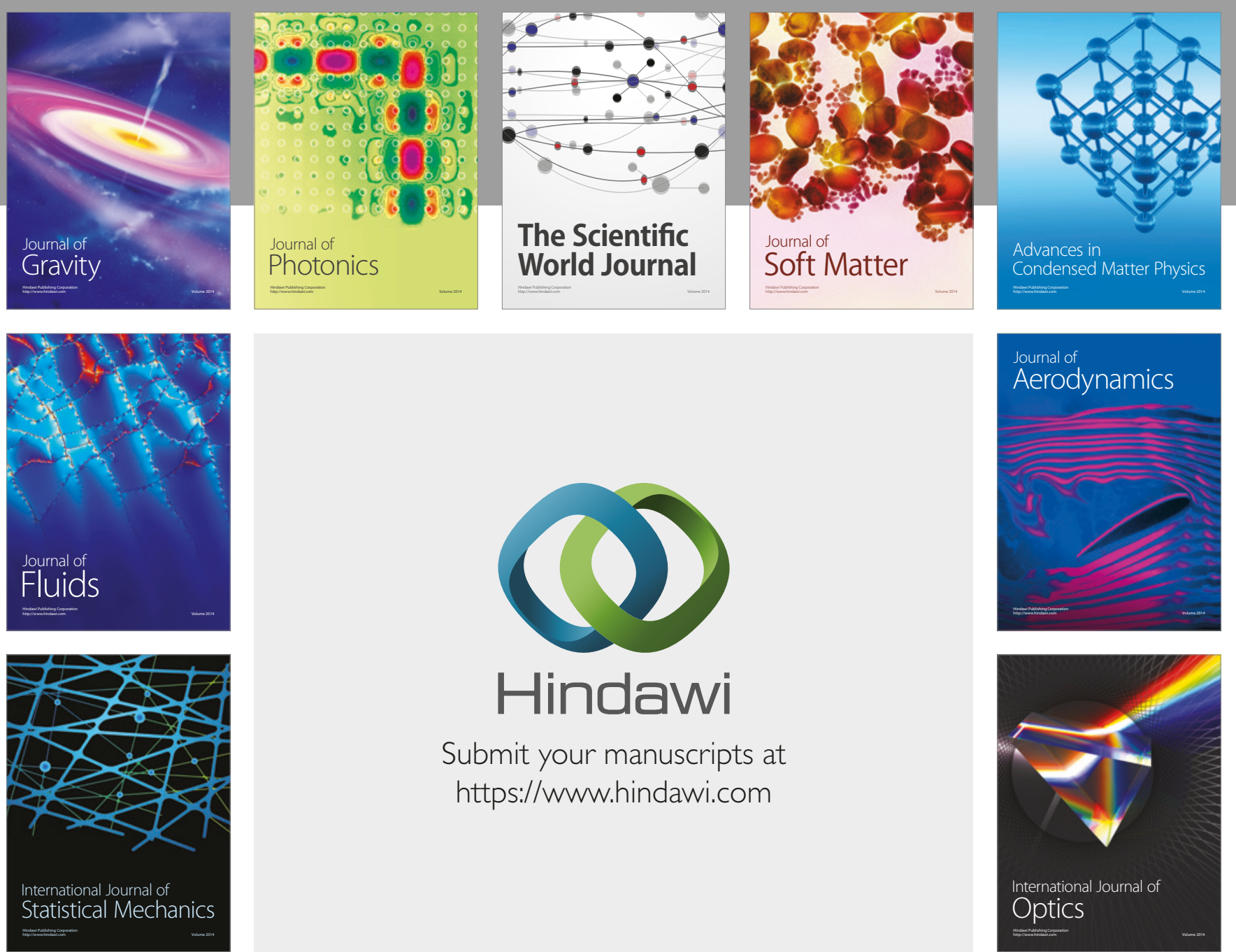

Submit your manuscripts at

https://www.hindawi.com
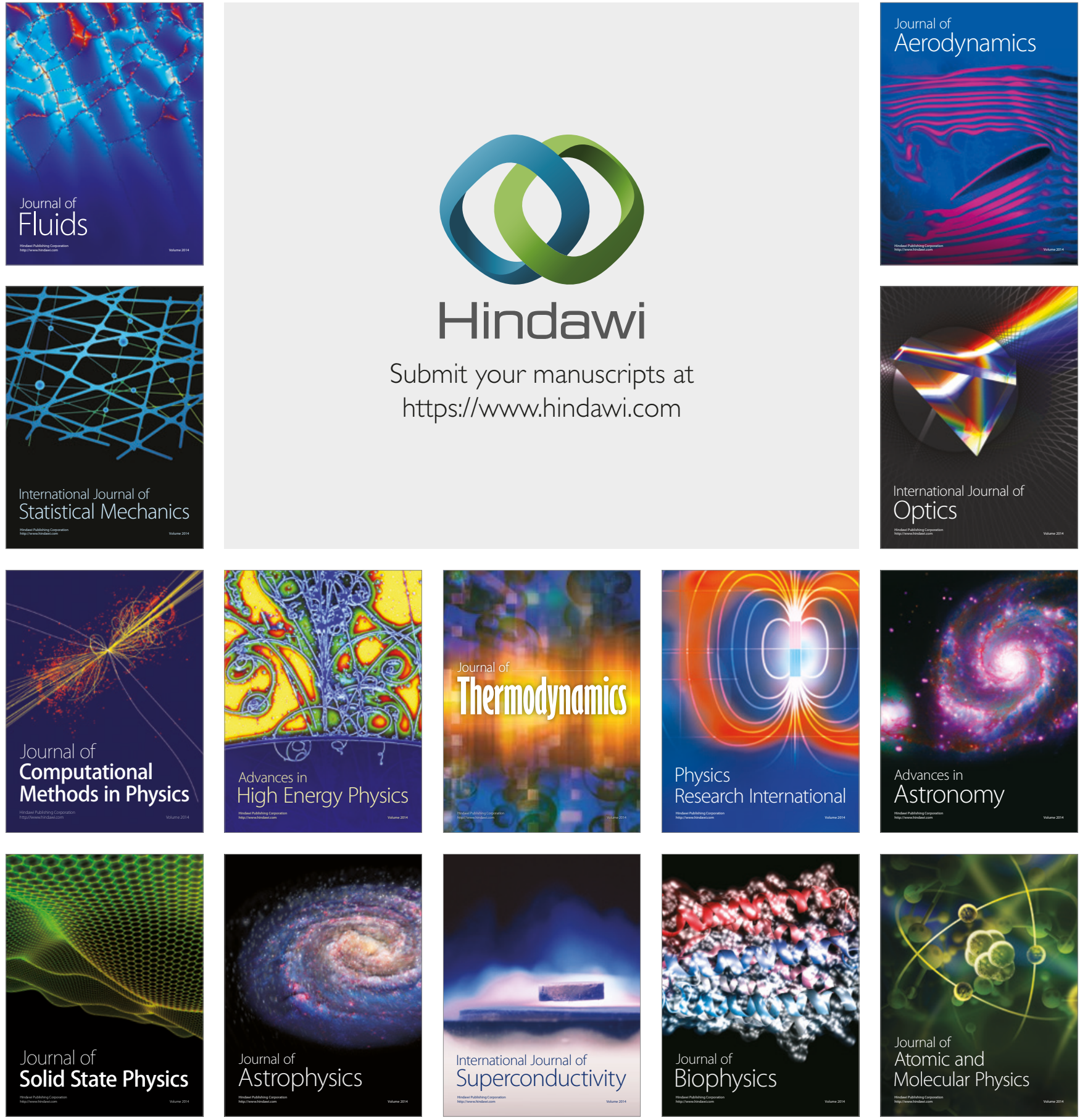